\title{
Effets immunomodulateurs des macrolides au cours des pathologies respiratoires chroniques
}

\author{
Macrolides as immunomodulatory drugs in chronic lung diseases
}

\section{A. Guillon · Y. Jouan · A. Petit · F. Gueugnon}

Reçu le 2 août 2012 ; accepté le 5 décembre 2012

(C) SRLF et Springer-Verlag France 2012

Résumé Les macrolides ont une variété de propriétés physiologiques autres que leur seule activité antibiotique qui a justifié leur développement initial. L'ensemble de ces propriétés est généralement regroupé sous le nom d'immunomodulation. Ce spectre d'action s'étend à la régulation d'action leucocytaire et la production de médiateurs inflammatoires, au contrôle de la sécrétion de mucus et à la modulation des mécanismes de défense de l'épithélium respiratoire. Ils ont également des propriétés antivirales et semblent pouvoir inhiber la formation de biofilm et la synthèse de facteurs de virulence de Pseudomonas aeruginosa. Ces propriétés sont généralement observées à des faibles doses et pour des temps d'exposition longs. L'intérêt des macrolides pour le traitement des affections respiratoires chroniques est croissant, principalement pour les atteintes inflammatoires marquées par des phases d'exacerbation, déclenchées le plus souvent par un stimulus infectieux. Leur bénéfice sur la prévention d'exacerbation dans la bronchopneumopathie chronique obstructive et la mucoviscidose est maintenant bien démontré. Leur utilisation dans l'asthme en traitement de fond n'a pas fait la preuve de son efficacité. Dans cette mise au point, nous nous proposons de revenir sur les propriétés immunomodulatrices des macrolides pour mieux comprendre les mécanismes d'action soutenant leur utilisation dans les atteintes respiratoires chroniques inflammatoires, et nous synthétisons les principales investigations cliniques de leurs utilisations dans la bronchopneumopathie chronique obstructive, la mucoviscidose et l'asthme.

A. Guillon $(\bowtie) \cdot$ Y. Jouan · F. Gueugnon

Université François Rabelais, EA 6305, F-37032 Tours, France

e-mail : antoine.guillon@univ-tours.fr

A. Guillon · A. Petit · F. Gueugnon

INSERM, Centre d'étude des pathologies respiratoires,

UMR 1100/EA6305, F-37032 Tours, France

\section{A. Guillon · Y. Jouan}

Service de réanimation médicale,

CHRU Bretonneau, 2 boulevard Tonnellé,

F-37044 Tours cedex 9
Mots clés Macrolide · Immunomodulation · Épithélium respiratoire $\cdot$ Bronchopneumopathie chronique obstructive $\cdot$ Mucoviscidose

\begin{abstract}
Macrolides have a variety of physiological activities other than antimicrobial effects. The non-antimicrobial properties of macrolides are grouped under the concept of immunomodulation. The spectrum of action of these antibiotics extends to regulation of leukocyte function and production of inflammatory mediators, control of mucus production, and modulation of respiratory epithelial defense. In addition, macrolides present anti-viral effects and are able to inhibit biofilm formation and reduce production of virulence factors from Pseudomonas aeruginosa. These properties of macrolides are mainly observed at low doses and for long-term treatments. There is a growing interest for the use of these drugs as immunomodulatory therapy for chronic lung diseases, mainly inflammatory diseases with acute exacerbation triggered by infections. The clinical benefits of macrolides to prevent acute exacerbation in chronic obstructive pulmonary disease and cystic fibrosis are well demonstrated. On the other side, there is no evidence that macrolides are efficient in asthma. Here, we review the physiological activities of macrolides including mechanisms by which they contribute to the clinical effects observed in chronic lung diseases. We also present the main clinical investigations regarding the use of macrolides in chronic obstructive pulmonary disease, cystic fibrosis, and asthma.
\end{abstract}

Keywords Macrolide · Immunomodulation - Respiratory epithelium $\cdot$ Chronic obstructive pulmonary disease $\cdot$ Cystic fibrosis

\section{Introduction}

Les macrolides ont été développés pour leur capacité à inhiber la synthèse protéique bactérienne : ils empêchent 
l'élongation de la chaîne peptidique en se fixant à la sousunité $50 \mathrm{~S}$ des ribosomes bactériens. Ils sont intrinsèquement actifs contre les bactéries Gram $(+)$, quelques bactéries Gram (-), ainsi que les germes atypiques. Cependant, ils ont également de nombreuses propriétés non antibiotiques. Leur capacité à moduler des fonctions cellulaires, ainsi que leurs actions immunomodulatrices sont mieux comprises actuellement. Leur champ d'utilisation est donc en train de s'élargir, principalement vers les pathologies chroniques respiratoires.

\section{Propriétés anti-inflammatoires et immunomodulatrices des macrolides sur les voies respiratoires lors d'interactions hôtes-pathogènes}

Les principales propriétés anti-inflammatoires et immunomodulatrices des macrolides sur les voies respiratoires sont synthétisées sur la Figure 1.

\section{Mécanismes d'action sur l'hôte}

\section{Effets sur les macrophages alvéolaires}

En plus de la fonction de phagocytose des pathogènes, les macrophages alvéolaires jouent un rôle fondamental dans la phagocytose des cellules apoptotiques. Ils permettent ainsi d'éviter une nécrose secondaire, qui induirait une inflamma- tion locale. Dans la bronchopneumopathie chronique obstructive (BPCO), l'apoptose des cellules épithéliales est augmentée et les fonctions phagocytaires des macrophages sont altérées. Il a été montré que l'azithromycine pouvait stimuler les fonctions phagocytaires des macrophages de patients BPCO [1-3]. Par ailleurs, les macrolides activent la différentiation des monocytes en macrophages [4-6]. In vitro [7] et dans un modèle animal d'infection à Pseudomonas aeurginosa [8], l'azithromycine modifie le phénotype des macrophages alvéolaires vers un phénotype d'activation alternative (Alternatively Activated Macrophages) aux propriétés anti-inflammatoires et dont les fonctions sont orientées vers l'efferocytose (phagocytose des cellules apoptotiques), le nettoyage des débris cellulaires, le remodelage et la réparation tissulaire après agression $[9,10]$.

\section{Effets sur le mucus}

Le mucus est sécrété par les cellules épithéliales et est composé d'eau, de mucines, de phospholipides du surfactant et de protéines et peptides à activité antimicrobienne. Il participe donc activement à la défense des voies aériennes. Dans de nombreuses maladies respiratoires telles que la BPCO ou la mucoviscidose, on observe une sécrétion de mucus anormale, en termes de composition et/ou de propriétés physicochimiques. Ces changements altèrent la clairance mucociliaire, ce qui favorise les infections chroniques et l'inflammation [11]. De nombreuses données suggèrent que les macrolides diminuent l'hypersécrétion de mucus,

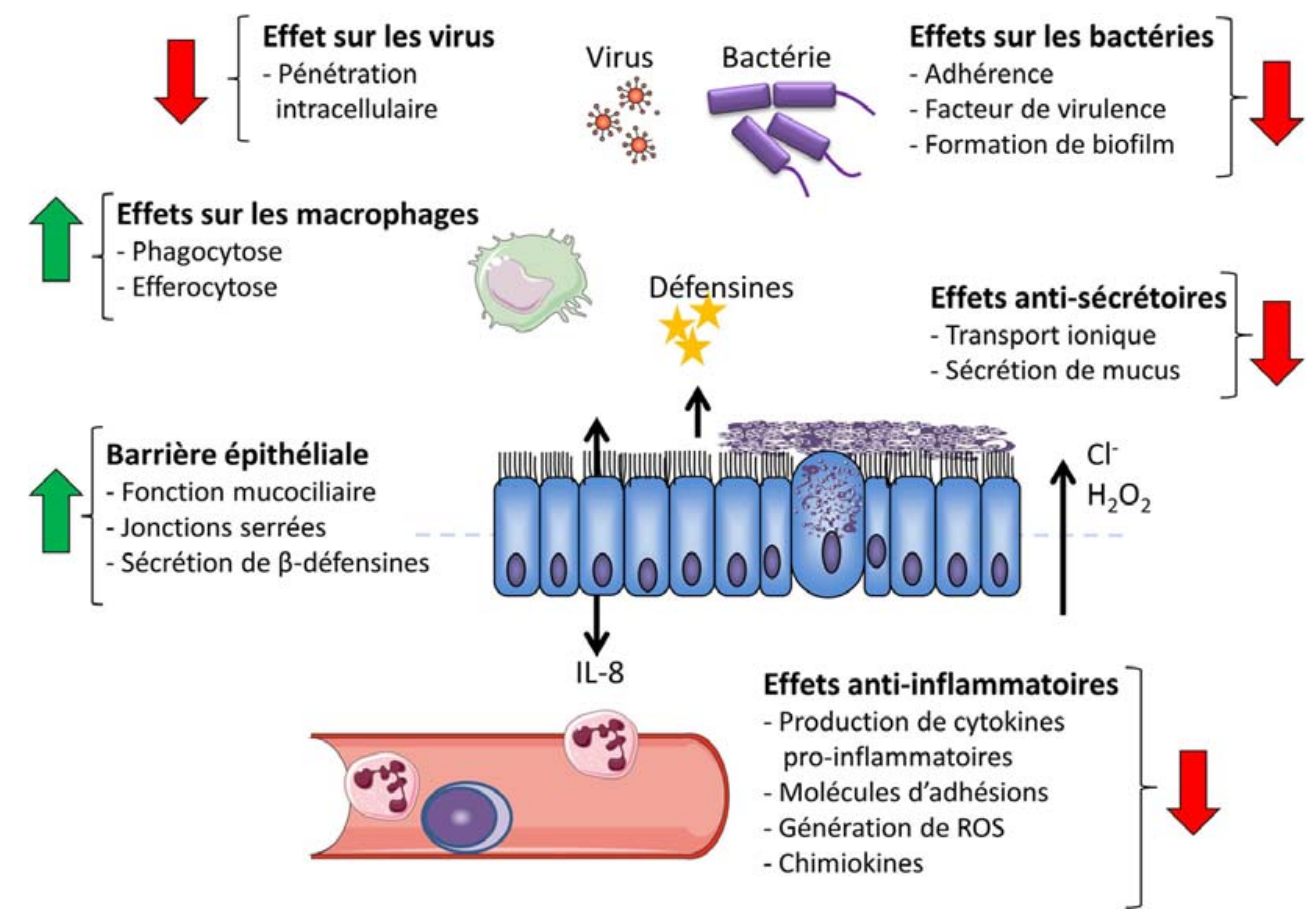

Fig. 1 Synthèse des potentiels effets bénéfiques des macrolides sur l'épithélium respiratoire humain en phase inflammatoire 
notamment par leur action régulatrice sur l'expression des mucines. Dans un modèle expérimental d'épithélium nasal de rats sensibilisé à l'ovalbumine, il a été montré que la clarithromycine inhibait significativement la sécrétion de mucus et l'infiltration de neutrophiles induite par l'instillation d'ovalbumine ou de lipopolysaccharide (LPS) [12]. Ces résultats ont ensuite été confirmés sur des cultures de cellules d'épithélium respiratoire humain, où un traitement par macrolide a entrâné une diminution de la production de MUC5AC (glycoprotéine essentielle de la phase gel du mucus) en réponse à un stimulus inflammatoire. Le mécanisme d'action n'en est pas complètement connu. Un modèle murin de panbronchiolite diffuse a permis de montrer que la clarithromycine inhibait la surproduction de la mucine 5 ac induite par l'infection avec $P$. aeruginosa, en inhibant la phosphorylation des protéines Extracellular signal-regulated kinase (ERK) [13]. De même, la roxithromycine inhibait l'hypersécrétion de mucus induite notamment par le LPS via une inhibition de l'activation et de la translocation nucléaire du nuclear factor-kappa $B$ (NF-kB), facteur de transcription impliqué dans la réponse au stress cellulaire, qui est luimême un effecteur en aval dans la voie ERK $[14,15]$. Enfin, un autre mécanisme par lequel les macrolides exerceraient leurs effets bénéfiques sur l'hypersécrétion de mucus serait lié à leur capacité à bloquer l'influx calcique, notamment dans les glandes submucosales, via une activation partielle des récepteurs muscariniques à l'acétylcholine [16].

Ces données expérimentales ont été confirmées chez l'homme à la suite de plusieurs investigations cliniques. Il a été montré que l'administration de clarithromycine réduisait significativement le volume des expectorations et modifiait leur composition chez environ $40 \%$ des patients souffrant de bronchite chronique ou bronchiectasie [17]. De même, dans un autre essai clinique, la clarithromycine (100 mg x 2/jour pendant huit semaines) réduisait la production de mucus de moitié chez des patients atteints de panbronchiolite diffuse, de bronchite chronique ou de bronchectasie [18]. Le traitement modifiait également la rhéologie du mucus qui devenait plus élastique. Il n'avait aucun impact sur la flore microbienne retrouvée dans les expectorations (quantité de bactérie et diversité).

\section{Effets sur les cellules épithéliales}

La barrière épithéliale est la première ligne de défense contre l'agression des pathogènes, et par conséquent la première à subir les dommages. Des études in vitro d'agression épithéliale retrouvent un effet protecteur des macrolides face à une simulation d'agression bactérienne [19-21], possiblement par stabilisation des membranes des cellules épithéliales. En plus de la fonction de barrière physique, les cellules épithéliales ont également un rôle actif dans la défense immunitaire, et l'élimination de microorganismes présents dans les voies aériennes.
Les cellules épithéliales ont notamment la propriété de sécréter des peptides à activité antimicrobienne à très large spectre (Anti-Microbial Peptides - AMP). Les défensines sont les AMP les plus étudiés, ils détruisent les agents pathogènes principalement par perméabilisation membranaire. Ils peuvent être exprimés de façon constitutive (ex : $\beta$-défensines-1), ou bien être induits par un stimulus inflammatoire (ex : $\beta$-défensines-2). Leur déficit est une des causes explorées dans les pathologies respiratoires chroniques avec des phases d'exacerbations infectieuses : déficit inné (ex : mucoviscidose) ou acquis (ex : exposition à la fumée de cigarette). Les effets des macrolides sur cette sécrétion d'AMP par les cellules épithéliales ont été étudiés expérimentalement. Il a été montré que le liquide de surface de l'épithélium respiratoire avait un pouvoir bactéricide supérieur lorsque le modèle cellulaire utilisé était préalablement traité avec de faibles doses d'érythromycine, alors même que les germes étaient résistants aux macrolides (P. aeruginosa, $S$. aureus avec concentration minimale inhibitrice $[\mathrm{CMI}]>4 \mu \mathrm{g} / \mathrm{ml}$ ) [22]. Il a ensuite été précisé que l'érythromycine, comme l'azitromycine, augmentaient l'expression d'acide ribonucléique messager (ARNm) codant pour les $\beta$-défensines - 1 et -2 . L'augmentation effective de la sécrétion protéique des $\beta$-défensines 1 et 2 a également été confirmée par western blot [22]. Ces données suggèrent que les macrolides sont capables d'augmenter le pouvoir bactéricide du liquide de surface des voies aériennes, en partie grâce à l'induction de $\beta$-défensines par les cellules épithéliales respiratoires.

\section{Effets sur les mouvements ioniques}

L'épithélium bronchique est connu pour réguler la teneur en électrolytes du liquide de surface des voies respiratoires. Le volume de ce liquide de surface est régulé par les mouvements d'eau découlant notamment de l'influx de sodium et de l'efflux de chlore à travers l'épithélium. Dans la mucoviscidose, la déficience du canal chlore CFTR entraine un déficit en chlore extracellulaire, ce qui se traduit par une rétention d'eau accrue et donc une augmentation de la viscosité du mucus, réduisant ainsi la clairance mucociliaire, le tout formant un terrain propice aux infections [23].

En ce qui concerne les effets des macrolides sur l'efflux des ions $\mathrm{Cl}^{-}$, les données de la littérature sont controversées. En 2005, il a été montré que ni la clarithromycine, ni l'azithromycine ne modifiaient les caractéristiques du transport ionique de l'épithélium nasal normal et muté pour CFTR chez l'homme et la souris [24]. À l'inverse, des données plus récentes obtenues in vitro suggèrent que l'azithromycine augmenterait l'efflux de $\mathrm{Cl}^{-}$des cellules épithéliales bronchiques humaines mutées pour le gène CFTR, mais pas des cellules «normales » $[25,26]$. Cette augmentation de l'efflux des ions chlorures pourrait être liée à une action des macrolides sur le canal CFTR lui-même, celle-ci étant abolie par l'utilisation d'inhibiteurs spécifiques. Puisque la composition en électrolytes 
du liquide de surface peut affecter la défense des poumons, la régulation $\mathrm{du}$ transport ionique via le système paracellulaire pourrait jouer un rôle important dans la prévention des infections pulmonaires. Ce transport ionique paracellulaire est contrôlé par des protéines transmembranaires formant les jonctions serrées basolatérales : claudines, occludines et molécules de jonctions cellulaires JAMs. L'importance de ces protéines dans le maintien de l'homéostasie ionique a été démontrée par l'existence de maladies génétiques récessives comme l'hypomagnésémie rénale (liée une mutation de la claudine 16) ou encore certaines formes de surdité dans lesquelles la mutation de la claudine 14 perturbe l'environnement ionique du conduit cochléaire [27,28]. Il a été suggéré que le fonctionnement des jonctions serrées pouvait être altéré en cas d'inflammation chronique. Coyne et al. ont montré que l'exposition de cellules bronchiques à certaines cytokines pro-inflammatoires dont les taux sont accrus chez les patients atteints de mucoviscidose, entraînait une diminution de la résistance transépithéliale, une augmentation de la perméabilité paracellulaire et une modification de la morphologie des jonctions serrées [29]. Une autre étude menée avec des cellules primaires bronchiques humaines a révélé que certains macrolides comme l'azithromycine pouvaient augmenter la résistance transépithéliale et provoquer un réarrangement des claudines 1 et 4 ainsi que de l'occludine et JAM-A vers le compartiment intracellulaire [30]. Ce résultat est à mettre en parallèle avec l'observation de Lee et al. qui montre que $P$. aeruginosa se fixe plus facilement sur les épithéliums ayant une faible résistance transépithéliale [31]. Ainsi, cet effet particulier de l'azithromycine pourrait en partie expliquer l'efficacité de ce macrolide dans le traitement de la mucoviscidose.

\section{Effets sur les médiateurs inflammatoires}

Les cytokines et les chimiokines sont des molécules clés de l'initiation, la perpétuation et la résolution de la réponse inflammatoire. Il est classiquement admis que certaines sont proinflammatoires (interleukine [IL]-1, IL-6, IL-8, Tumor necrosing factor [TNF]- $\alpha$ ) et d'autres anti-inflammatoires (IL-10). Les macrolides vont globalement diminuer la production de cytokines pro-inflammatoires [32], mais à la différence des immunosuppresseurs comme les corticoïdes, les macrolides sont bien immunomodulateurs, entrainnant une régulation non linéaire de la production de cytokines. Il a en effet été montré que la production d'IL- 8 en réponse à une induction par LPS en présence de macrolide était multiphasique [33] avec une phase initiale de down-regulation, puis d'up-regulation transitoire et enfin un retour au taux basal du contrôle. Des réponses similaires ont également été retrouvées in vivo pour l'IL-1 et l'IL-2 [34]. De même, in vivo, l'azithromycine active initialement les leucocytes de sujets sains, puis induit secondairement une inhibition du burst oxydatif et de la production de cytokines proinflammatoires [35].

\section{Effets sur les polynucléaires neutrophiles}

Les polynucléaires neutrophiles sont des cellules clés de la défense immunitaire innée du poumon, rapidement et massivement recrutées en cas d'invasion de pathogènes. Les macrolides ont des actions multiples sur les polynucléaires :

- Chimiotactisme : le recrutement des polynucléaires neutrophiles au niveau pulmonaire est largement dépendant de la sécrétion locale de chimiokines. En présence de macrolides, la production de ces chimiokines attractantes est diminuée, notamment l'IL-8 [36,37]. Dans de nombreuses situations cliniques d'inflammation pulmonaire chronique, il est effectivement retrouvé une diminution du compte de polynucléaires neutrophiles dans les secrétions trachéobronchiques ou alvéolaires après traitement par macrolides [38-41]. Toutes les études ne sont pas concordantes, notamment celles portant sur la BPCO [42-44], mais il existe de nombreuses limites dans l'extrapolation des données de ces études pilotes étudiant des macrolides différents, avec des durées d'exposition différentes et sur des pathologies inflammatoires chroniques de gravité variable ;

- adhésion : à proximité des sites inflammatoires, les cellules endothéliales qui ont été activées par les médiateurs pro-inflammatoires vont exprimer sur leur versant luminal des molécules d'adhésion. Les polynucléaires en transit vont ainsi se lier aux cellules endothéliales par une série de molécules transmembranaires d'adhésion jusqu'à traverser l'endothélium pour parvenir au site inflammatoire. La roxithromycine [45] et l'érythromycine [46,47] agissent sur Mac-1, une molécule d'adhésion des polynucléaires neutrophiles, en inhibant sa production et donc de ce fait le recrutement des polynucléaires ;

- dégranulation : plusieurs données in vitro $[48,49]$ suggèrent que la dégranulation des polynucléaires est diminuée en présence de macrolides. L'implication clinique n'en est cependant pas évaluée à ce jour ;

- burst oxydatif et production d'espèces réactives de l'oxygène : les polynucléaires neutrophiles ont la capacité de synthétiser rapidement des espèces réactives de l'oxygène (Reactive Oxygen Species [ROS]) par une consommation brutale d'oxygène et l'utilisation de la nicotinamide adénine dinucléotide phosphate (NADPH) oxydase. Les ROS sont extrêmement réactifs et peuvent modifier et altérer des molécules, autant de propriétés utilisées dans la défense antimicrobiennes. Les données concernant l'effet des macrolides à ce niveau sont également encore divergentes et sujettes à caution : certaines études retrouvent une augmentation de la production de ROS en présence de macrolides [50], alors que d'autres retrouvent une diminution $[51,52]$. Les différences observées sont peutêtre secondaires à des variations des conditions expérimentales. Cependant, il est également possible qu'il existe 
une réponse non linéaire à l'exposition aux macrolides en termes de production de ROS et/ou une réponse dépendant de la concentration intracellulaire en macrolide [53]. De plus, l'effet des ROS ne se limite pas à l'activité antimicrobienne : ils sont également impliqués dans la signalisation cellulaire [54]. Il est donc trop simpliste de considérer que seule une baisse de la production de ROS a un effet immunomodulateur ;

- apoptose : après recrutement et activation, les polynucléaires vont normalement s'engager vers l'apoptose durant la phase de résolution de l'inflammation. En situation de pathologie inflammatoire chronique pulmonaire, le recrutement et l'activation persistent. L'apoptose des polynucléaires activés n'est plus optimale. Ils meurent donc par nécrose et aggravent encore l'inflammation locale. Les macrolides semblent induire l'apoptose des polynucléaires activés $[55,56]$ et contribueraient de ce fait à la résolution de l'inflammation.

\section{Mécanismes d'action sur les pathogènes respiratoires}

\section{Bactéries}

Les macrolides n'ont pas d'activité antibiotique contre le $P$. aeruginosa. Pourtant, dans un modèle de souris bactériémique à $P$. aeruginosa, de faibles doses d'erythromycine améliorent la survie de 20 à $80 \%$ [57]. Plusieurs mécanismes d'action des macrolides sur $P$. aeruginosa ont été explorés (voir revue [58]). Tout d'abord, il a été montré in vitro que de faibles doses d'érythromycine réduisaient leur pouvoir d'adhésion et la synthèse de facteurs de virulence. Ensuite, lorsque les conditions de survie sont défavorables, $P$. aeruginosa a la capacité de modifier sa morphologie externe vers un phénotype « biofilm ». Cette transformation peut être inhibée par les macrolides, via l'inhibition de la synthèse d'alginate, un composant essentiel à la cohésion du biofilm. Enfin, il existe un mécanisme régulateur qui coordonne l'expression de certains gènes bactériens, au sein d'une même population bactérienne. Cette communication de cellules à cellules est nommée la détection du quorum. Il semblerait que les macrolides puissent entraver cette détection du quorum $[58,59]$.

\section{Virus}

Depuis que les propriétés anti-inflammatoires et immunomodulatrices des macrolides ont été décrites, l'intérêt de ces molécules a également été testé dans le cadre des pathologies virales respiratoires. Il est en effet connu que la morbimortalité induite par les virus respiratoires est en partie liée à l'orage cytokinique et à la dérégulation de l'inflammation secondaire à l'infection virale. Les propriétés inhibitrices des macrolides sur la production d'IL-6 et d'IL-8 par les cellules épithéliales respiratoires sont donc intéressantes dans ce contexte. De façon plus concrète, il a été montré que l'ajout de clarithromycine sur des cultures de lignées cellulaires (A549) infectées à rhinovirus (RV) diminuait la concentration du virus dans le surnageant [60]. Étonnamment, l'inhibition de la réplication virale par la clarithromycine était plus marquée avec des doses faibles de macrolides, et lorsque l'antibiotique était administré avant l'infection. Le virus, pour se répliquer, pénètre dans les cellules épithéliales respiratoires en se liant à un récepteur de surface cellulaire, l'intercellular adhesion molecule-1 (ICAM-1). ICAM-1 est une protéine de surface constitutive, mais dont l'expression est augmentée par des effecteurs inflammatoires et également par le RV. Il a été démontré que l'érythromycine et la clarithromycine diminuent l'induction d'ICAM-1, bloquant ainsi l'entrée d'ARN viral dans la cellule et donc la réplication virale $[60,61]$. Il est probable que cette diminution d'induction d'ICAM-1 par les macrolides soit la conséquence de leurs propriétés anti-inflammatoires ou immunomodulatrices. En effet, les macrolides inhibent la production d'IL-1 $\beta$ et IL-8, qui sont des inducteurs d'ICAM-1. De plus, il a également été démontré que l'érythromycine pouvait inhiber in vitro l'infection par le virus respiratoire syncytial selon un principe superposable au RV [62].

\section{Applications cliniques dans les pathologies respiratoires chroniques}

\section{Bronchopneumopathie chronique obstructive}

La BPCO est actuellement un enjeu de santé majeur. Elle concerne 3,5 millions de personne en France et sa prévalence est estimée à 24 millions aux États-Unis. L'Organisation Mondiale de la santé estime d'ailleurs que la BPCO sera la $3^{\mathrm{e}}$ cause de décès dans le monde en 2030. Les phases d'exacerbation sont déclenchées le plus souvent par des infections, bactériennes ou virales, et ont un impact majeur sur la morbimortalité des patients. Pour leurs propriétés antiinflammatoires et immunomodulatrices, en plus de leurs propriétés antimicrobiennes, les macrolides ont été testés comme traitements préventifs des phases d'exacerbations chez les patients BPCO (Tableau 1). Plusieurs investigations cliniques ont été menées, avec des résultats jusqu'à présent partagés. Initialement, il avait été montré un effet protecteur des macrolides (érythromycine) sur la fréquence des exacerbations et des hospitalisations chez des patients BPCO à l'état sable [63]. Cependant, ce résultat n'avait ensuite pas été confirmé : la prise orale de clarithromycine pendant trois mois chez des patients BPCO stables n'avait pas d'effet sur la fréquence des exacerbations [44]. En 2008, Seemungal et al. [64] rapportaient également qu'un traitement quotidien par des faibles doses d'érythromycine réduisait la fréquence des exacerbations chez des patients avec une BPCO modérée 


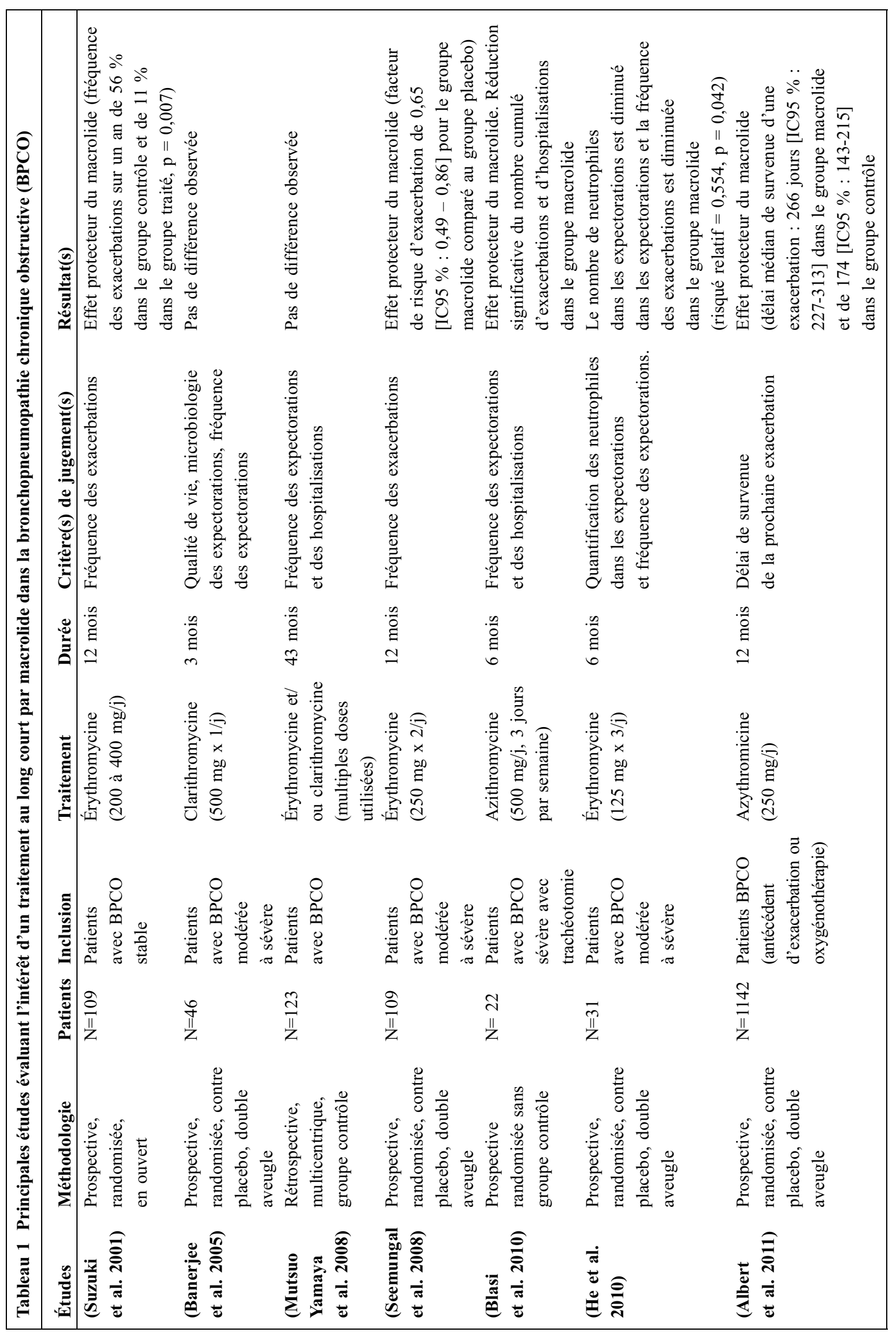


à sévère : sur les 12 mois de l'étude, la fréquence médiane (intervalle de confiance $95 \%$, IC95\%) des exacerbations était de 1,00 (0,00-2,00) dans le groupe macrolide et de 2,00 $(0,25-3,75)$ dans le groupe placebo [64]. Dans cette étude, aucune différence n'avait été observée dans l'analyse microbiologique des expectorations et dans l'analyse des marqueurs inflammatoires (IL-6 et IL-8 dans les expectorations, protéine C-réactive et IL-6 dans le sérum). Enfin, une étude de plus grande envergure a récemment considérablement augmenté le niveau de preuve du bénéfice d'un traitement au long court par un macrolide pour prévenir les phases d'exacerbation [65]. Un total de 1142 patients BPCO a été randomisé en double aveugle pour recevoir un placebo ou un traitement quotidien par $250 \mathrm{mg}$ d'azithromycine sur une durée d'un an. Le délai médian de la première exacerbation était de 266 jours (IC95 \% : 227-313) dans le groupe macrolide et de 174 (IC95 \% : 143-215) dans le groupe contrôle. Il est peu probable que ce résultat soit attribuable aux propriétés antibactériennes de l'azithromycine compte tenu des faibles doses employées, et des échecs précédents des autres classes d'antibiotiques. Cette étude a permis cette fois de clore le débat sur l'efficacité des macrolides : un traitement au long terme par une faible dose de macrolide permet de réduire la fréquence des exacerbations de BPCO ayant un antécédent d'exacerbation ou un traitement par oxygénothérapie [66]. Par contre, la pertinence d'adopter cette stratégie à plus grande échelle reste à établir, notamment en termes de pression de sélection microbienne chez ces patients dont les voies aériennes sont souvent colonisées par des agents pathogènes, mais également en termes d'effets secondaires cardiovasculaires [67].

\section{Mucoviscidose}

L'intérêt des propriétés immunomodulatrices des macrolides est connu depuis plus longtemps dans la mucoviscidose (Tableau 2). En 2002, deux études prospectives, randomisées vs placebo, ont rapporté un effet bénéfique d'un traitement au long court par l'azithromycine sur les performances respiratoires de patients atteints de mucoviscidose $[68,69]$. Ces résultats ont été confirmés en 2003 par une étude multicentrique portant sur 185 patients atteints de mucoviscidose et chroniquement infectés par $P$. aeruginosa [70]. Ces patients étaient randomisés et recevaient un placebo ou de l'azithromycine ( 250 à $500 \mathrm{mg} / \mathrm{j}$ selon le poids, trois jours par semaine, pendant 24 semaines). Le nombre d'exacerbation a été significativement réduit dans le groupe macrolide. Le volume expiratoire maximal par seconde (VEMS) a augmenté de 4,4\% (exprimé en pourcentage prédit sur la valeur théorique) dans le groupe macrolide sur cette période alors qu'il a décliné de $1,8 \%$ dans le groupe placebo $(p=0,001)$. Fort de ces résultats, les auteurs de ce premier travail ont conduit une deuxième étude, en incluant des patients atteints de mucoviscidose non infectés par $P$. aeruginosa [71]. Dans cette deuxième population de patients, le traitement par azithromycine n'a pas eu d'effet significatif sur le VEMS, cependant la fréquence des exacerbations était réduite de moitié dans le groupe macrolide. Cette stratégie thérapeutique pose également la question de l'effet d'une exposition prolongée à des antibiotiques sur l'évolution des résistances microbiennes, chez des patients souvent colonisés par de multiples pathogènes. Dans l'étude de Saiman et al. en 2010 [71], le traitement par macrolide n'avait pas modifié le profil microbiologique des expectorations, mais les résistances de $S$. aureus et de $H$. influenzae ont augmenté dans le groupe macrolide. Aucune transmission de $S$. aureus résistant au macrolide n'a été observée dans les personnes contact des sujets traités au long court par macrolide [72].

\section{Asthme}

L'asthme est également une pathologie chronique inflammatoire respiratoire pour laquelle des traitements au long court par macrolide ont été testés. Un nombre important d'investigations cliniques ont été publiées, mais avec des effectifs de patients souvent réduits et des durées de traitement courtes, ce qui rend leur interprétation difficile. Une méta-analyse publiée en 2007 fait état de 98 études sur ce sujet dont sept finalement analysées (416 patients avec un asthme chronique) [73]. Il n'a pas été observé de différence significative sur le VEMS, ni sur la consommation de corticoïdes. Le niveau de preuve scientifique est donc actuellement insuffisant pour statuer sur la place des macrolides dans l'arsenal thérapeutique permettant le contrôle de l'asthme. Les macrolides pourront peut-être avoir un rôle comme thérapeutique adjuvante dans certains phénotypes de la maladie. Par exemple, un traitement par clarithromycine a été testé pendant huit semaines en double aveugle et contre placebo chez 45 patients ayant un asthme sévère réfractaire [38]. Il a été montré une réduction significative de la sécrétion d'IL-8 et du nombre de neutrophile dans les voies respiratoires, ainsi qu'une diminution de l'activité de l'élastase et de la matrix métalloprotéinase 9, suggérant une régulation globale de l'activation des neutrophiles chez ces patients par la clarithromycine.

\section{Conclusion}

Les macrolides ont des activités biologiques bien plus larges que les propriétés antimicrobiennes qui ont permis leur développement, il y a plus de 50 ans. Leurs effets sont variés et s'observent à différents niveaux de l'épithélium respiratoire, mais convergent globalement dans le sens d'une immunomodulation. Leurs mécanismes d'action sont encore imparfaitement connus. Il est probable que la modification de voie(s) de signalisation(s), en amont de la cascade inflammatoire, ne soit 


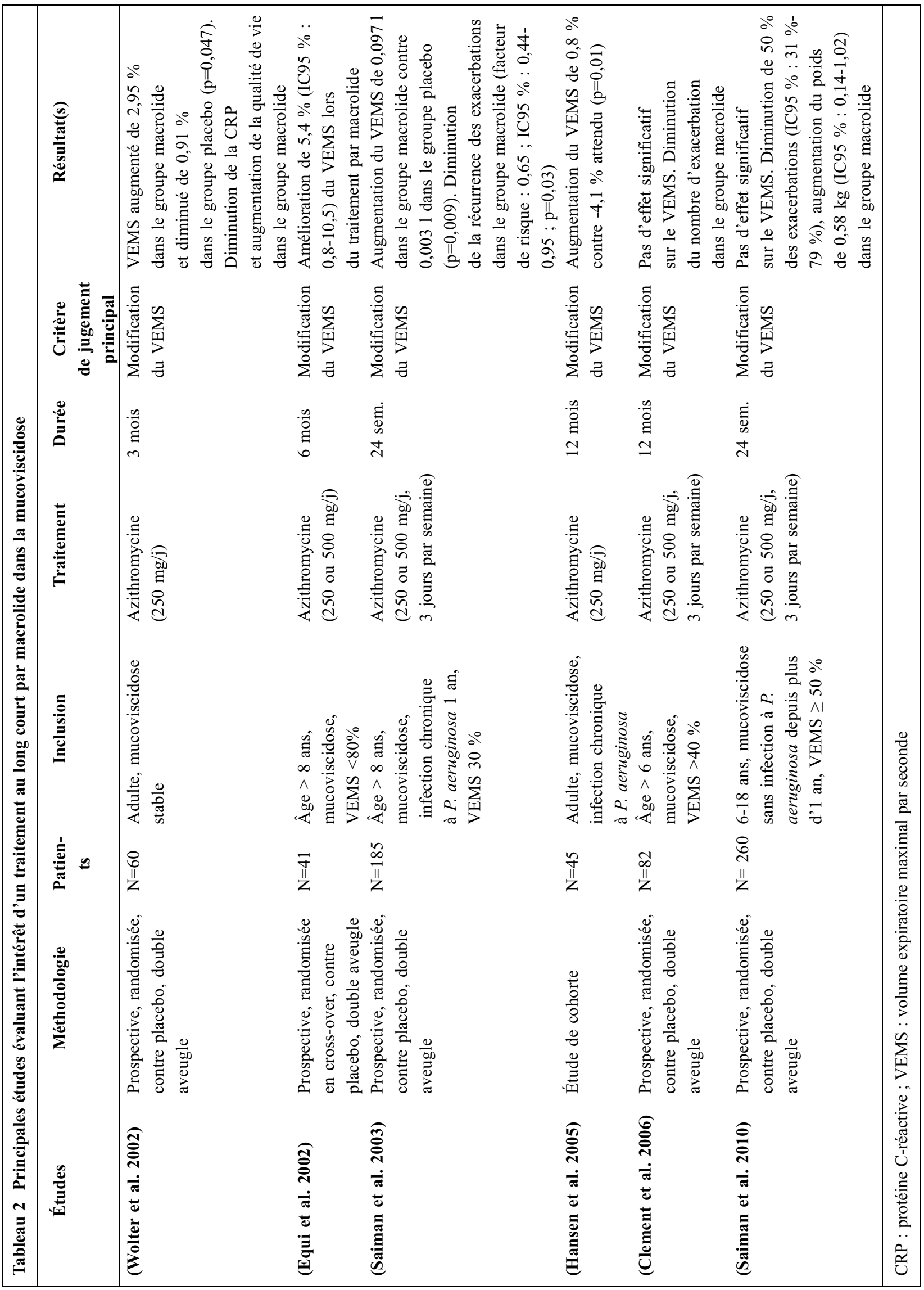


pas encore identifiée. Une meilleure connaissance de ces mécanismes d'action et de leurs cibles cellulaires permettrait de contribuer au développement de molécules aux propriétés anti-inflammatoires différentes des médicaments actuellement disponibles. Ainsi, des molécules dérivées de la classe des macrolides, mais sans activité antibactérienne, sont en cours de synthèse [74,75]. Les études précédemment décrites seront donc probablement à refaire pour déterminer si les propriétés anti-inflammatoires ou immunomodulatrices (à condition qu'elles soient conservées) sont bien responsables des effets observés. Enfin, il semblerait plus confortable d'utiliser une molécule n'exerçant pas de pression de sélection microbienne, compte tenu de la pénurie actuelle de nouvelles classes antibiotiques.

Remerciements : les auteurs remercient Thomas Baranek pour sa participation dans la conception des figures.

Conflit d'intérêt : les auteurs déclarent ne pas avoir de conflit d'intérêt.

\section{Références}

1. Hodge S, Hodge G, Brozyna S, et al (2006) Azithromycin increases phagocytosis of apoptotic bronchial epithelial cells by alveolar macrophages. Eur Respir J 28:486-95

2. Hodge S, Hodge G, Jersmann H, et al (2008) Azithromycin Improves Macrophage Phagocytic Function and Expression of Mannose Receptor in Chronic Obstructive Pulmonary Disease. Am J Respir Crit Care Med 178:139-48

3. Hodge S, Reynolds PN (2012) Low-dose azithromycin improves phagocytosis of bacteria by both alveolar and monocyte-derived macrophagesin chronic obstructive pulmonary disease subjects. Respirology 17:802-7

4. Keicho N, Kudoh S, Yotsumoto H, et al (1994) Erythromycin promotes monocyte to macrophage differentiation. J Antibiot 47:80-9

5. Sunazuka T, Yoshida K, Oohori M, et al (2003) Effect of 14-membered macrolide compounds on monocyte to macrophage differentiation. J Antibiot 56:721-4

6. Yoshida K, Sunazuka T, Nagai K, et al (2005) Macrolides with Promotive Activity of Monocyte to Macrophage Differentiation. J Antibiot (Tokyo) 58:79-81

7. Murphy BS, Sundareshan V, Cory TJ, et al (2008) Azithromycin alters macrophage phenotype. J Antimicrob Chemother 61:554-60

8. Feola DJ, Garvy BA, Cory TJ, et al (2010) Azithromycin Alters Macrophage Phenotype and Pulmonary Compartmentalization during Lung Infection with Pseudomonas. Antimicrob Agents Chemother 54:2437-47

9. Varin A, Gordon S (2009) Alternative activation of macrophages: Immune function and cellular biology. Immunobiology 214:630-41

10. Gordon S (2003) Alternative activation of macrophages. Nat Rev Immunol 3:23-5

11. Randell SH, Boucher RC (2006) Effective mucus clearance is essential for respiratory health. Am J Respir Cell Mol Biol 35:20-8

12. Shimizu T, Shimizu S, Hattori R, et al (2003) In vivo and in vitro effects of macrolide antibiotics on mucus secretion in airway epithelial cells. Am J Respir Crit Care Med 168:581-7
13. Kaneko Y, Yanagihara K, Seki M, et al (2003) Clarithromycin inhibits overproduction of muc5ac core protein in murine model of diffuse panbronchiolitis. Am J Physiol Lung Cell Mol Physiol 285:L847-53

14. Ou XM, Feng YL, Wen FQ, et al (2008) Macrolides attenuate mucus hypersecretion in rat airways through inactivation of NF-kappaB. Respirology 13:63-72

15. Kim DY, Takeuchi K, Ishinaga H, et al (2004) Roxithromycin suppresses mucin gene expression in epithelial cells. Pharmacology $72: 6-11$

16. Lu S, Liu H, Farley JM Sr (2011) Macrolide antibiotics inhibit mucus secretion and calcium entry in Swine airway submucosal mucous gland cells. J Pharmacol Exp Ther 336:178-87

17. Tagaya E, Tamaoki J, Kondo M, et al (2002) Effect of a short course of clarithromycin therapy on sputum production in patients with chronic airway hypersecretion. Chest 122:213-8

18. Tamaoki J, Takeyama K, Tagaya E, et al (1995) Effect of clarithromycin on sputum production and its rheological properties in chronic respiratory tract infections. Antimicrob Agents Chemother 39:1688-90

19. Anderson R, Theron AJ, Feldman C (1996) Membrane-stabilizing, anti-inflammatory interactions of macrolides with human neutrophils. Inflammation 20:693-705

20. Feldman C, Anderson R, Theron AJ, et al (1997) Roxithromycin, clarithromycin, and azithromycin attenuate the injurious effects of bioactive phospholipids on human respiratory epithelium in vitro. Inflammation 21:655-65

21. Nakano T, Ohashi Y, Tanaka A, et al (1998) Roxythromycin reinforces epithelial defence function in rabbit trachea. Acta Otolaryngol Suppl 538:233-8

22. Ishizawa K, Suzuki T, Yamaya M, et al (2005) Erythromycin increases bactericidal activity of surface liquid in human airway epithelial cells. Am J Physiol Lung Cell Mol Physiol 289:L565-73

23. Smith JJ, Travis SM, Greenberg EP, et al (1996) Cystic fibrosis airway epithelia fail to kill bacteria because of abnormal airway surface fluid. Cell 85:229-36

24. Barker PM, Gillie DJ, Schechter MS, et al (2005) Effect of macrolides on in vivo ion transport across cystic fibrosis nasal epithelium. Am J Respir Crit Care Med 171:868-71

25. Oliynyk I, Varelogianni G, Schalling M, et al (2009) Azithromycin increases chloride efflux from cystic fibrosis airway epithelial cells. Exp Lung Res 35:210-21

26. Saint-Criq V, Rebeyrol C, Ruffin M, et al (2011) Restoration of chloride efflux by azithromycin in airway epithelial cells of cystic fibrosis patients. Antimicrob Agents Chemother 55:1792-3

27. Simon DB, Lu Y, Choate KA, et al (1999) Paracellin-1, a renal tight junction protein required for paracellular $\mathrm{Mg} 2+$ resorption. Science 285:103-6

28. Wilcox ER, Burton QL, Naz S, et al (2001) Mutations in the gene encoding tight junction claudin-14 cause autosomal recessive deafness DFNB29. Cell 104:165-72

29. Coyne CB, Vanhook MK, Gambling TM, et al (2002) Regulation of airway tight junctions by proinflammatory cytokines. Mol Biol Cell 13:3218-34

30. Asgrimsson V, Gudjonsson T, Gudmundsson GH, et al (2006) Novel effects of azithromycin on tight junction proteins in human airway epithelia. Antimicrob Agents Chemother 50:1805-12

31. Lee A, Chow D, Haus B, et al (1999) Airway epithelial tight junctions and binding and cytotoxicity of Pseudomonas aeruginosa. Am J Physiol 277:L204-17

32. Čulić O, Eraković V, Parnham MJ (2001) Anti-inflammatory effects of macrolide antibiotics. Eur J Pharmacol 429:209-29

33. Shinkai M, Foster GH, Rubin BK (2006) Macrolide antibiotics modulate ERK phosphorylation and IL-8 and GM-CSF production by human bronchial epithelial cells. Am J Physiol Lung Cell Mol Physiol 290:L75-L85 
34. Konno S, Adachi M, Asano K, et al (1992) Influences of roxithromycin on cell-mediated immune responses. Life Sci 51:PL107-12

35. Čulić O, Eraković V, Čepelak I, et al (2002) Azithromycin modulates neutrophil function and circulating inflammatory mediators in healthy human subjects. Eur J Pharmacol 450:277-89

36. Tsai WC, Rodriguez ML, Young KS, et al (2004) Azithromycin Blocks Neutrophil Recruitment in Pseudomonas Endobronchial Infection. Am J Respir Crit Care Med 170:1331-9

37. Tamaoki J, Nakata J, Tagaya E, et al (1996) Effects of roxithromycin and erythromycin on interleukin 8-induced neutrophil recruitment and goblet cell secretion in guinea pig tracheas. Antimicrobial Agents and Chemotherapy 40:1726

38. Simpson JL, Powell H, Boyle MJ, et al (2008) Clarithromycin Targets Neutrophilic Airway Inflammation in Refractory Asthma. Am J Respir Crit Care Med 177:148-55

39. Wilms EB, Touw DJ, Heijerman HG (2008) Pharmacokinetics and sputum penetration of azithromycin during once weekly dosing in cystic fibrosis patients. J Cyst Fibros 7:79-84

40. Verleden GM, Vanaudenaerde BM, Dupont LJ, et al (2006) Azithromycin Reduces Airway Neutrophilia and Interleukin-8 in Patients with Bronchiolitis Obliterans Syndrome. Am J Respir Crit Care Med 174:566-70

41. Yousaf N, Monteiro W, Parker D, et al (2010) Long-term lowdose erythromycin in patients with unexplained chronic cough: a double-blind placebo controlled trial. Thorax 65:1107-10

42. Parnham MJ, Čulić O, Eraković V, et al (2005) Modulation of neutrophil and inflammation markers in chronic obstructive pulmonary disease by short-term azithromycin treatment. Eur J Pharmacol 517:132-43

43. Basyigit I, Yildiz F, Ozkara SK, et al (2004) The Effect of Clarithromycin on Inflammatory Markers in Chronic Obstructive Pulmonary Disease: Preliminary Data. Ann Pharmacother 38:1400-5

44. Banerjee D, Khair OA, Honeybourne D (2005) The effect of oral clarithromycin on health status and sputum bacteriology in stable COPD. Respir Med 99:208-15

45. Kusano S, Kadota J, Kohno S, et al (1995) Effect of roxithromycin on peripheral neutrophil adhesion molecules in patients with chronic lower respiratory tract disease. Respiration 62:217-22

46. Sanz MJ, Nabah YN, Cerdá-Nicolás M, et al (2009) Erythromycin exerts in vivo anti-inflammatory activity downregulating cell adhesion molecule expression. Br J Pharmacol 144:190-201

47. Lin HC, Wang CH, Liu CY, et al (2000) Erythromycin inhibits $\beta 2$-integrins $(\mathrm{CD} 11 \mathrm{~b} / \mathrm{CD} 18)$ expression, interleukin-8 release and intracellular oxidative metabolism in neutrophils. Respiratory Medicine 94:654-60

48. Abdelghaffar H, Mtairag EM, Labro MT (1994) Effects of dirithromycin and erythromycylamine on human neutrophil degranulation. Antimicrob Agents Chemother 38:1548

49. Abdelghaffar H, Vazifeh D, Labro MT (1996) Comparison of various macrolides on stimulation of human neutrophil degranulation in vitro. J Antimicrob Chemother 38:81-93

50. Moutard I, Gressier B, Brunet C, et al (1998) In vitro interaction between spiramycin and polymorphonuclear neutrophils oxidative metabolism. Pharmacological Research 37:197-201

51. Labro MT, Benna J el, Babin-Chevaye C (1989) Comparison of the in-vitro effect of several macrolides on the oxidative burst of human neutrophils. J Antimicrob Chemother 24:561-72

52. Levert H, Gressier B, Moutard I, et al (1998) Azithromycin impact on neutrophil oxidative metabolism depends on exposure time. Inflammation 22:191-201

53. Levert H, Gressier B, Brunet C, et al (1999) Time and concentration dependent influence of dirithromycin on neutrophils oxidative burst. J Antibiot 52:127-33

54. Fang FC (2004) Antimicrobial reactive oxygen and nitrogen species: concepts and controversies. Nature Reviews Microbiology $2: 820-32$
55. Aoshiba K, Nagai A, Konno K (1995) Erythromycin shortens neutrophil survival by accelerating apoptosis. Antimicrob Agents Chemother 39:872-7

56. Inamura K, Ohta N, Fukase S, et al (2000) The effects of erythromycin on human peripheral neutrophil apoptosis. Rhinology 38:124-9

57. Hirakata Y, Kaku M, Tomono K, et al (1992) Efficacy of erythromycin lactobionate for treating Pseudomonas aeruginosa bacteremia in mice. Antimicrob Agents Chemother 36:1198-203

58. Kanoh S, Rubin BK (2010) Mechanisms of action and clinical application of macrolides as immunomodulatory medications. Clin Microbiol Rev 23:590-615

59. Delden C van, Köhler T, Brunner-Ferber F, et al (2012) Azithromycin to prevent Pseudomonas aeruginosa ventilator-associated pneumonia by inhibition of quorum sensing: a randomized controlled trial. Intensive Care Med 38:1118-25

60. Jang YJ, Kwon HJ, Lee BJ (2006) Effect of clarithromycin on rhinovirus-16 infection in A549 cells. Eur Respir J 27:12-9

61. Suzuki T, Yamaya M, Sekizawa K, et al (2002) Erythromycin inhibits rhinovirus infection in cultured human tracheal epithelial cells. Am J Respir Crit Care Med 165:1113-8

62. Asada M, Yoshida M, Suzuki T, et al (2009) Macrolide antibiotics inhibit respiratory syncytial virus infection in human airway epithelial cells. Antiviral Res 83:191-200

63. Suzuki T, Yanai M, Yamaya M, et al (2001) Erythromycin and common cold in COPD. Chest 120:730-3

64. Seemungal TA, Wilkinson TM, Hurst JR, et al (2008) Long-term erythromycin therapy is associated with decreased chronic obstructive pulmonary disease exacerbations. Am J Respir Crit Care Med 178:1139-47

65. Albert RK, Connett J, Bailey WC, et al (2011) Azithromycin for prevention of exacerbations of COPD. N Engl J Med 365:689-98

66. Wenzel RP, Fowler AA 3rd, Edmond MB (2012) Antibiotic Prevention of Acute Exacerbations of COPD. N Engl J Med 367:340-7

67. Ray WA, Murray KT, Hall K, et al (2012) Azithromycin and the risk of cardiovascular death. N Engl J Med 366:1881-90

68. Equi A, Balfour-Lynn IM, Bush A, et al (2002) Long term azithromycin in children with cystic fibrosis: a randomised, placebocontrolled crossover trial. Lancet 360:978-84

69. Wolter J, Seeney S, Bell S, et al (2002) Effect of long term treatment with azithromycin on disease parameters in cystic fibrosis: a randomised trial. Thorax 57:212-6

70. Saiman L, Marshall BC, Mayer-Hamblett N, et al (2003) Azithromycin in patients with cystic fibrosis chronically infected with Pseudomonas aeruginosa: a randomized controlled trial. JAMA 290:1749-56

71. Saiman L, Anstead M, Mayer-Hamblett N, et al (2010) Effect of azithromycin on pulmonary function in patients with cystic fibrosis uninfected with Pseudomonas aeruginosa: a randomized controlled trial. JAMA 303:1707-15

72. Tramper-Stranders GA, Ent CK van der, Gerritsen SA, et al (2007) Macrolide-resistant Staphylococcus aureus colonization in cystic fibrosis patients: is there transmission to household contacts? J Antimicrob Chemother 60:665-8

73. Richeldi L, Ferrara G, Fabbri LM, et al (2005) Macrolides for chronic asthma. Cochrane Database Syst Rev CD002997

74. Desaki M, Okazaki H, Sunazuka T, et al (2004) Molecular mechanisms of anti-inflammatory action of erythromycin in human bronchial epithelial cells: possible role in the signaling pathway that regulates nuclear factor-kappaB activation. Antimicrob Agents Chemother 48:1581-5

75. Mencarelli A, Distrutti E, Renga B, et al (2011) Development of non-antibiotic macrolide that corrects inflammation-driven immune dysfunction in models of inflammatory bowel diseases and arthritis. Eur J Pharmacol 665:29-39 